\title{
A self-healing polymer network with high strength, tunable properties and biocompatibility
}

\section{Supporting Information}

Broden Diggle ${ }^{1}$, Zhen Jiang ${ }^{1}$, Rachel W. Li $^{2}$, Luke A. Connal ${ }^{1 *}$

${ }^{1}$ Research School of Chemistry

Australian National University

Canberra, ACT 2601, Australia

${ }^{2}$ Medical School

Australian National University

Canberra, ACT 2601, Australia

*E-mail: luke.connal@anu.edu.au 
$\prod_{0}^{\mathrm{O}} \mathrm{H}_{7-8}^{\mathrm{O}}+\mathrm{H}_{\mathrm{OH}}^{\mathrm{O}}$
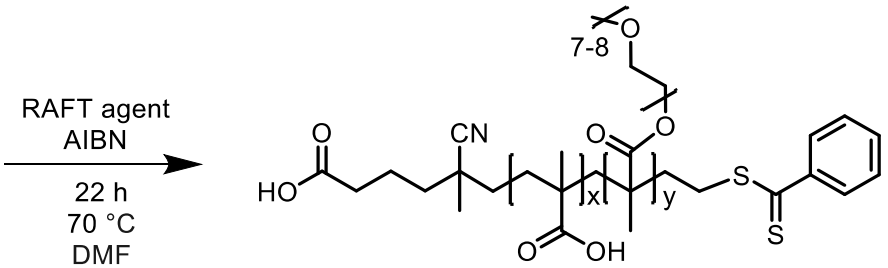

Scheme S1 - Schematic representation of the RAFT copolymerisation of OEGMA and MAAC, forming poly(MAAc-co-OEGMA).

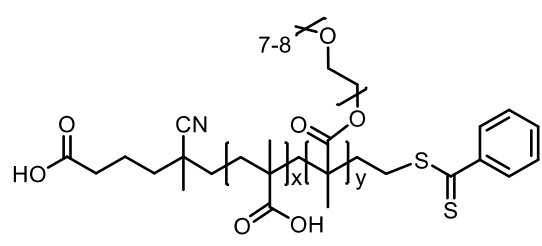<smiles>CC(C)[C@@H](C)CC(=O)c1ccc(O)cc1</smiles>

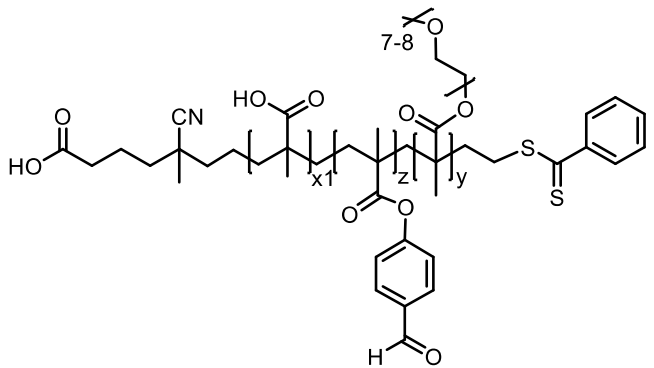

Scheme S2: Schematic representation of the aldehyde post-functionalisation of the co-polymer produced in scheme 1 using 4-hydroxybenzaldehyde to impart aldehyde moieties. 


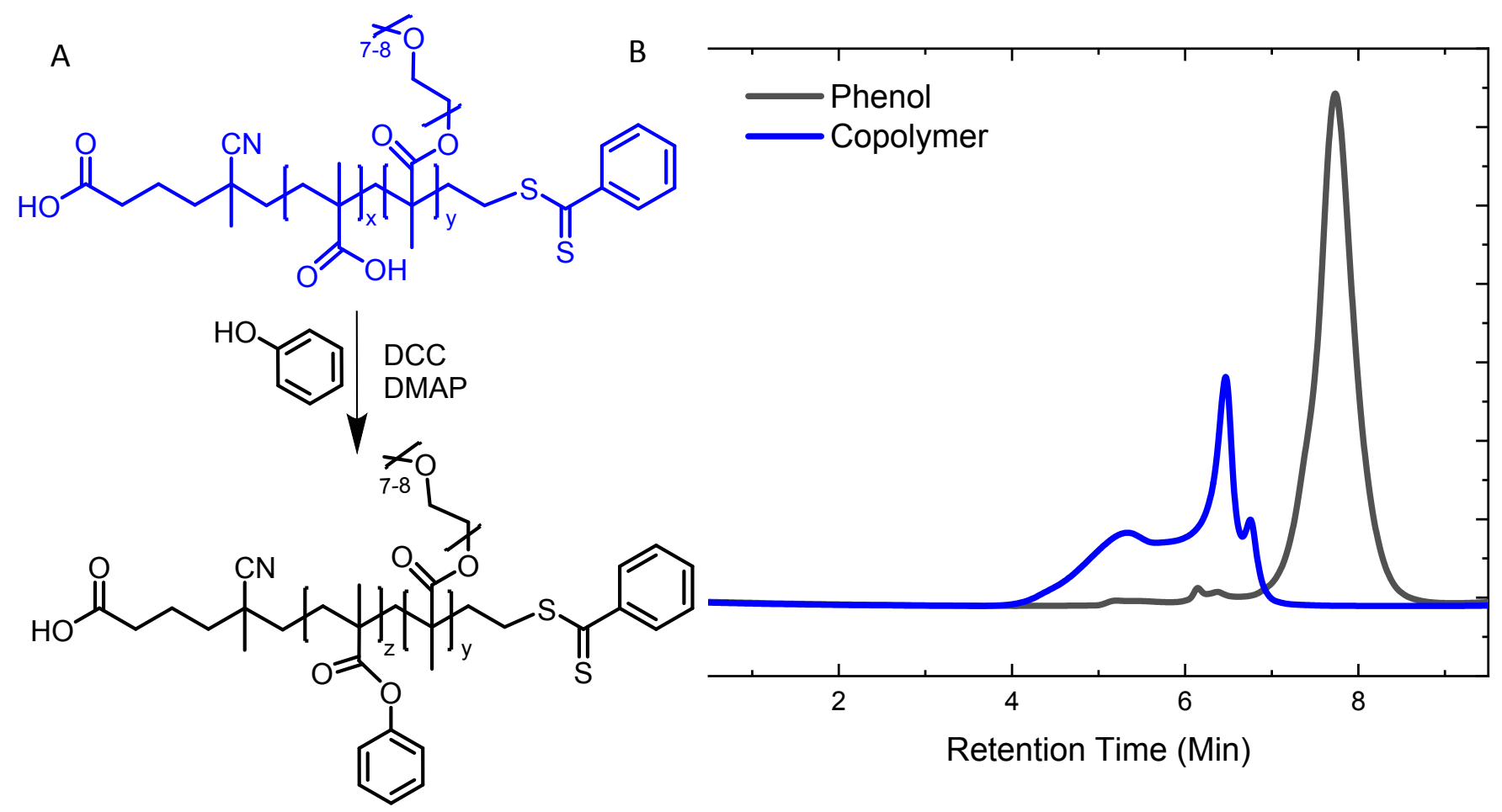

Figure S1: - The analytical result of modifying the molecular properties to effect macro properties. A: The reaction scheme for the esterification of the phenol and acid groups of MAAc. The reaction was completed with significant excess of phenol ( ${ }^{1} \mathrm{H} N M R$ Figure S6). The phenolisation in DMF, at room temperature for $30 \mathrm{~h}$ removed the significant hydrogen bonding, allowing for SEC analysis. B: SEC chromatograms of the copolymer (blue) and phenolised (black) copolymer. The copolymer ( $f e w$ million Da) had significant hydrogen bonding causing self-adhesion and additional interactions to the column compared to the phenolised chromatogram.

Qualitatively, the hydrogen bonding was reduced, as the isolated polymer was less sticky. The resultant estimated molecular weight from this modified system was 50,000 Da, meaning a typical postfunctionalised polymer would yield a molecular weight of around 40,000 Da. These results indicate the successful preparation of a polymer. 


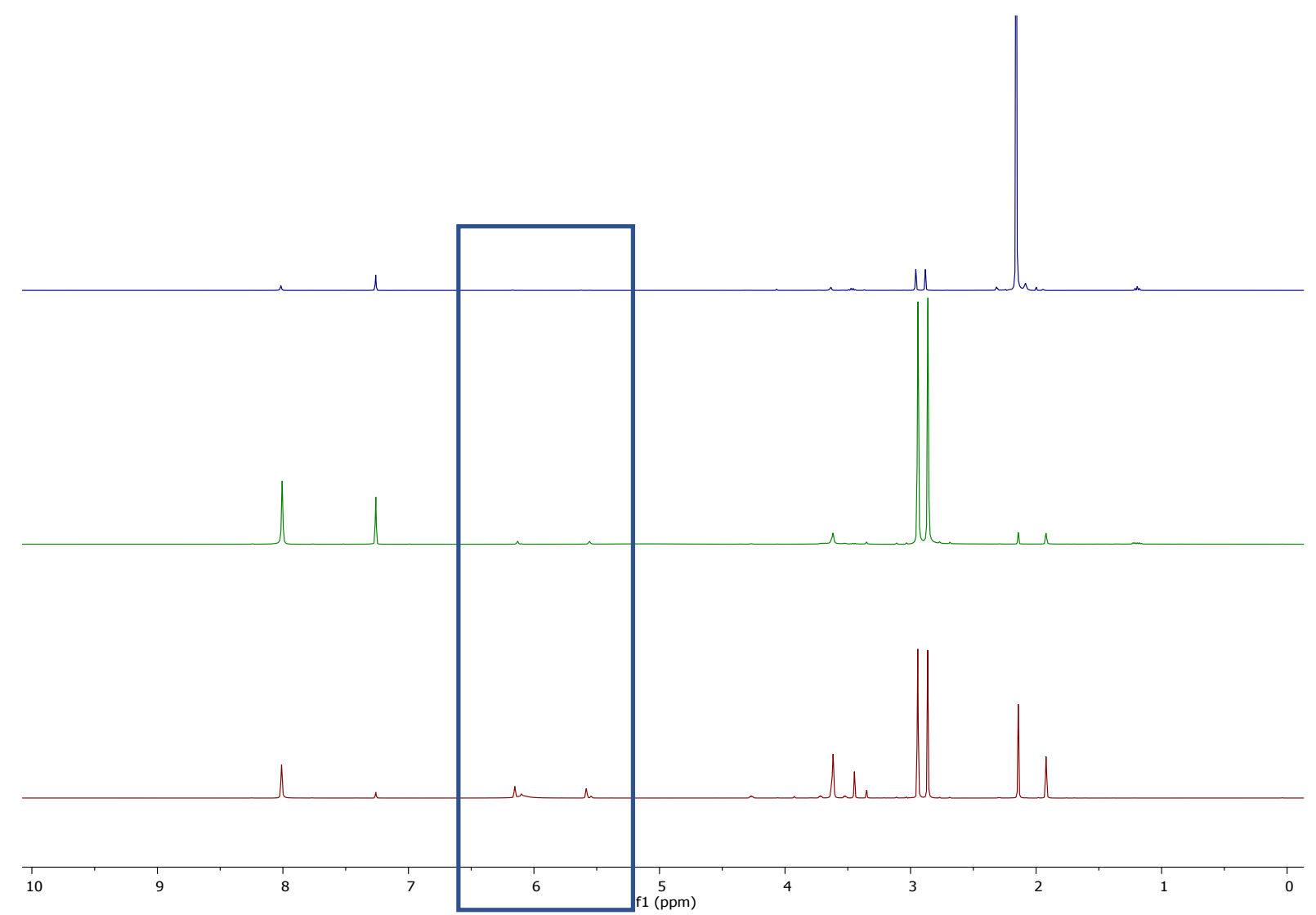

Figure S2: ${ }^{1} H$ NMR spectra monitoring the copolymer synthesis process. From the bottom to the top, it shows the unreacted solution, time $=0$ (bottom), the crude mixture (after $22 \mathrm{~h}$ at $70^{\circ} \mathrm{C}$, middle) and the purified product (top). The blue rectangular box highlights the removal of the monomer peaks. 

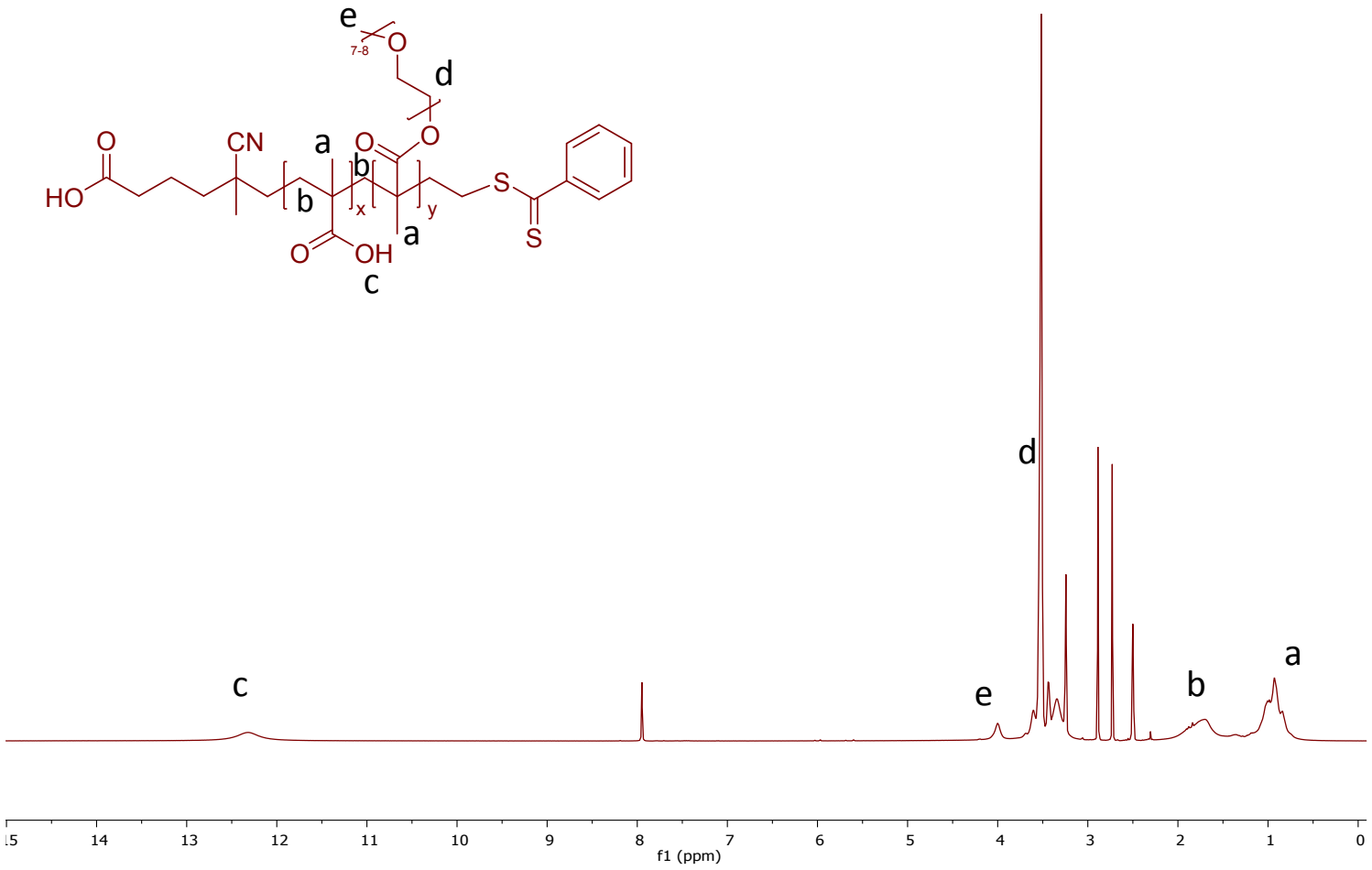

Figure S3: ${ }^{1} \mathrm{H}$ NMR of purified copolymer poly(MAAc-Co-OEGMA) with 85:15 feed ratio.

$$
\begin{aligned}
& 100 * \frac{\text { Integration }(e)}{3} / \frac{\text { Integration }(a)}{3}=O E G M A \%(a) \\
& \left(\frac{\text { Integration }(a)}{3}-\frac{\text { Integration }(e)}{3}\right) * 100=M A A c \% .(b)
\end{aligned}
$$

Equation S1: Formula for calculating the composition of the poly(MAAc-Co-OEGMA) copolymer. A) determines the OEGMA content by taking the methyl protons of OEGMA as a ratio of the total number of methyl protons (assuming RAFT agent is negligible). B) Then, taking the difference between the calculated OEGMA and the total methyl groups, results in the amount of MAAC.

Table S1 - Summary of compositions calculated from ${ }^{1} \mathrm{H}$ NMR analysis (Equation S1) compared to the feed ratios of copolymers synthesised.

\begin{tabular}{|l|l|l|}
\hline Polymer & $\begin{array}{l}\text { Molar Feed Ratio } \\
\text { (MAAc:OEGMA) }\end{array}$ & $\begin{array}{l}\text { 1H NMR calculated composition } \\
\text { (MAAc:OEGMA) }\end{array}$ \\
\hline 9010 & $90: 10$ & $90: 10$ \\
\hline 8515 & $85: 15$ & $86: 14$ \\
\hline 8515 PF & $86: 14$ & $60: 25: 15$ \\
\hline 7723 & $77: 23$ & $77: 23$ \\
\hline
\end{tabular}




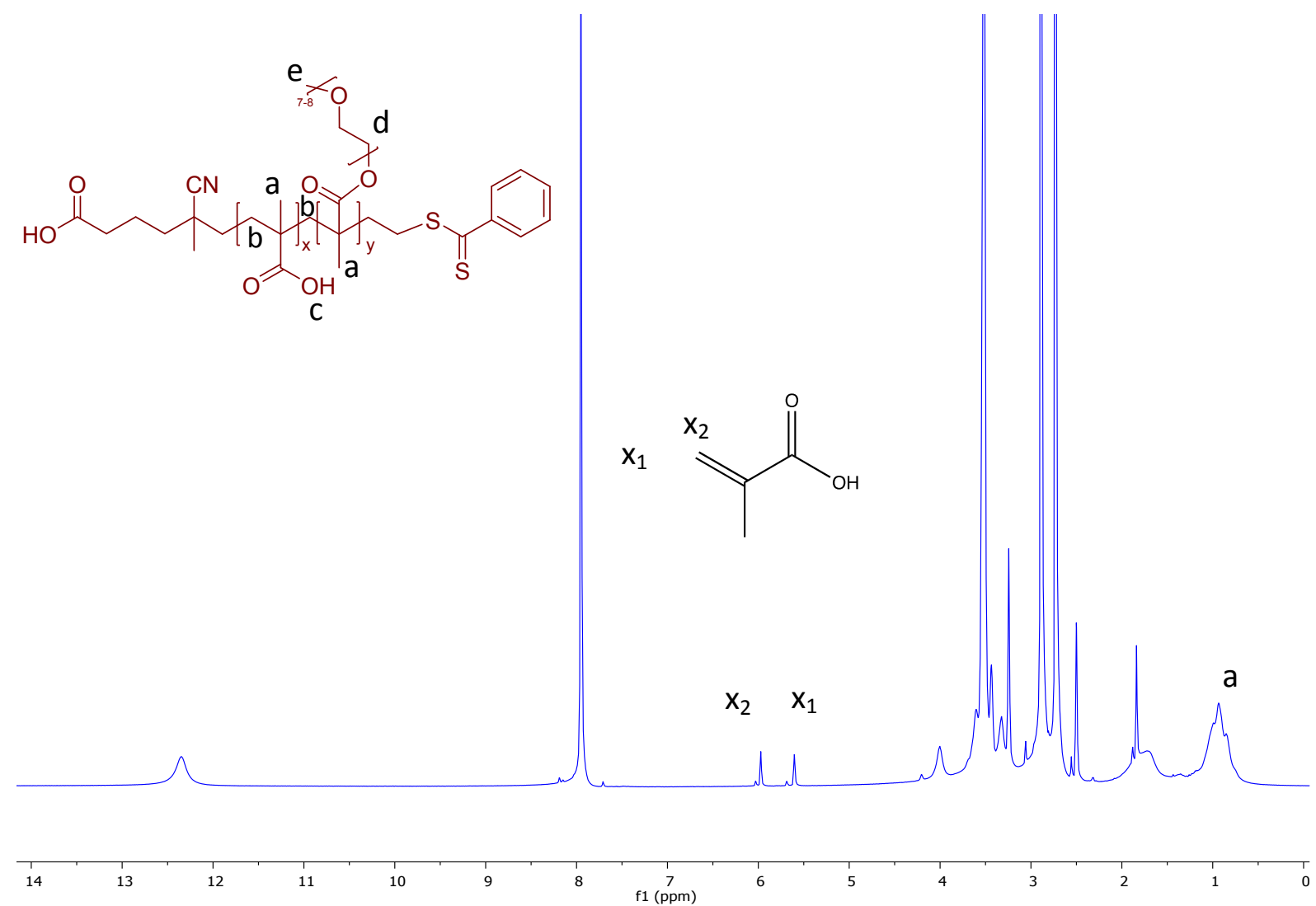

Figure S4: Crude ${ }^{1} H$ NMR of poly(MAAc-co-OEGMA). This spectrum has monomer peaks at 5.5 and 6 ppm $(x 1, x 2)$, which are used for Equation 2.

$$
\begin{aligned}
& \frac{\text { Integration }(a)}{3}-\frac{\text { monomer }(x 1)}{1}=\text { reaction ratio } \\
& \text { reaction ratio } / \frac{\text { Integration }(a)}{3}=\text { Conversion }(C)(b) \\
& M w= 2 * M A A c_{\text {feed }} * M w_{M A A C} * C+2 * O E G M A_{\text {feed }} * M w_{O E G M A} * C \\
& M w=2 * 85 * 86 * 0.9+2 * 15 * 500 * 0.9 \\
&\left.M w=26,500 \frac{g}{m o l}+R A F T \text { agent (negligible }\right)
\end{aligned}
$$

Molecular weight: 26,500 $\mathrm{g} \mathrm{mol}^{-1}$

Equation S2: Conversion of monomer to copolymer. A) Normalises the integrations between the methyl protons of the polymer and the monomer proton, subtracting the monomer amount from the methyl protons. B) Converts the amount from a) into a ratio; conversion approaches zero as time approaches zero, and approaches 1 as time progresses. C) Using the conversion to determine the approximate theoretical molecular weight using the feed ratios of the monomers. D) Putting in numbers for a copolymer with a feed ratio of 85:15. E) the total molecular weight. 


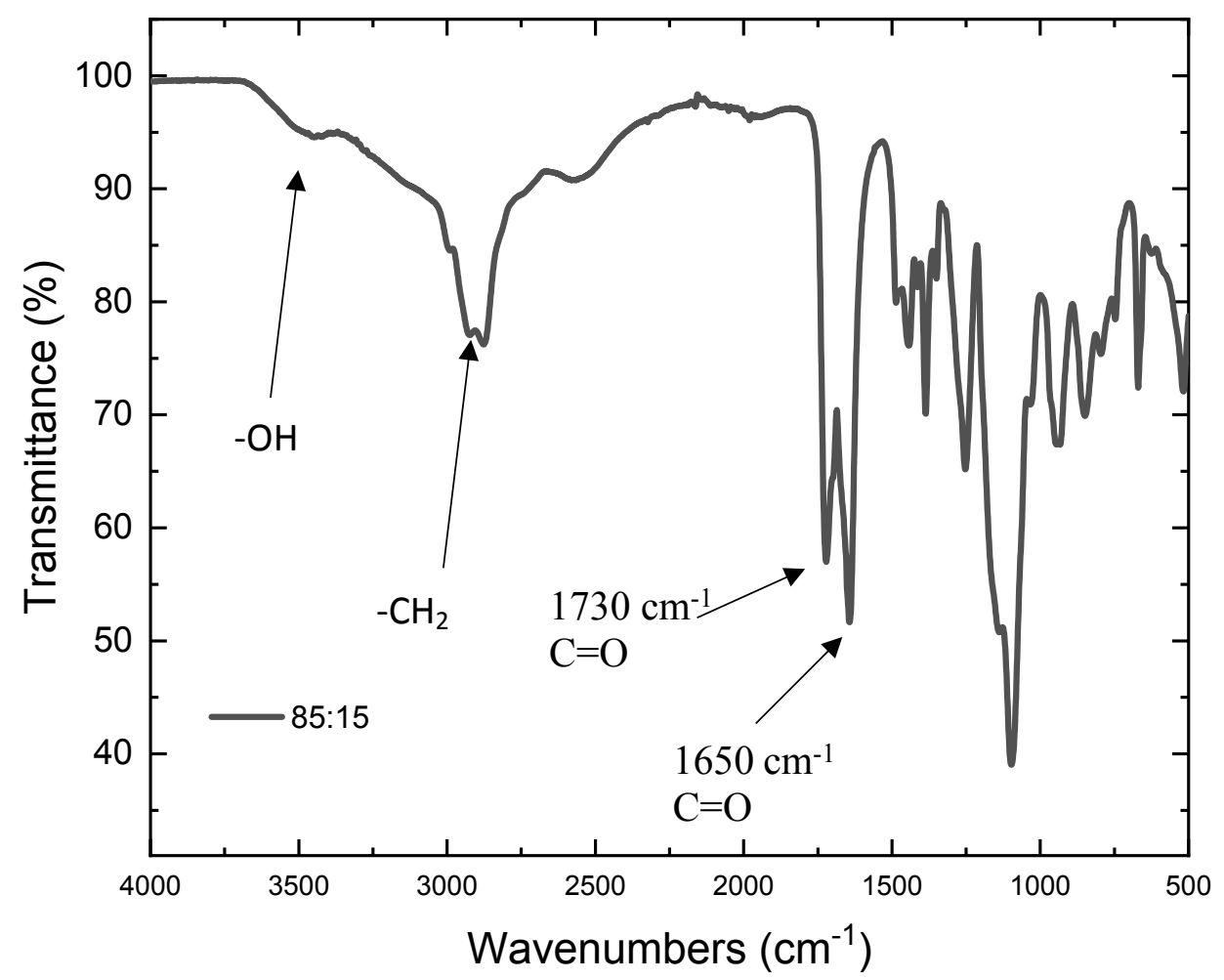

Figure S5: Complete FTIR spectrum of 85:15 copolymer poly $\left(M A A c_{85}-C 0-O E G M A_{15}\right)$ with key peaks labelled. 


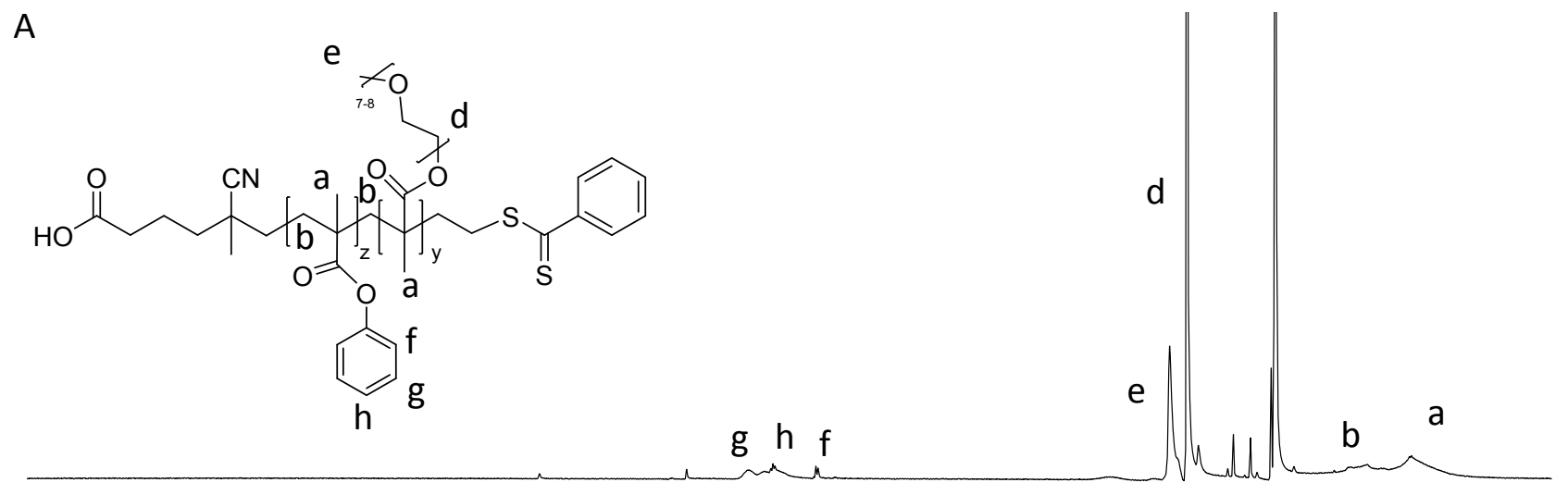

B
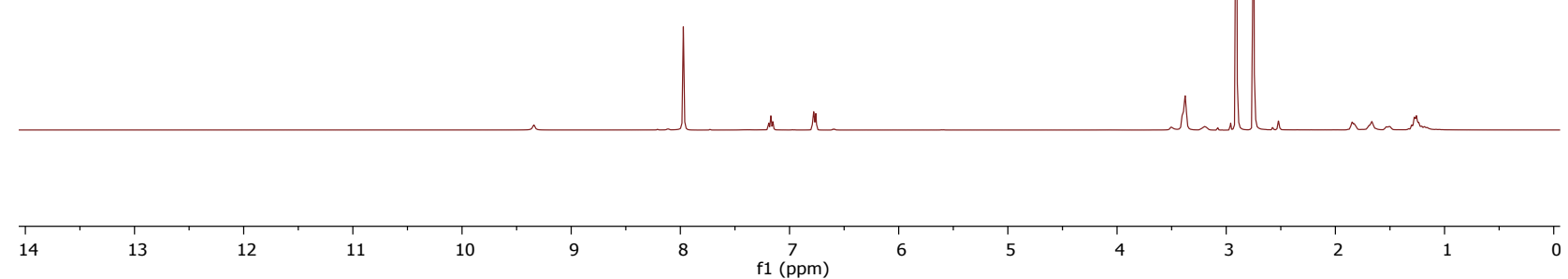

Figure S6: Purified $(A)$ and crude $(B){ }^{1} H$ NMR of the phenolisation of the copolymer, poly(MAAc-coOEGMA). 

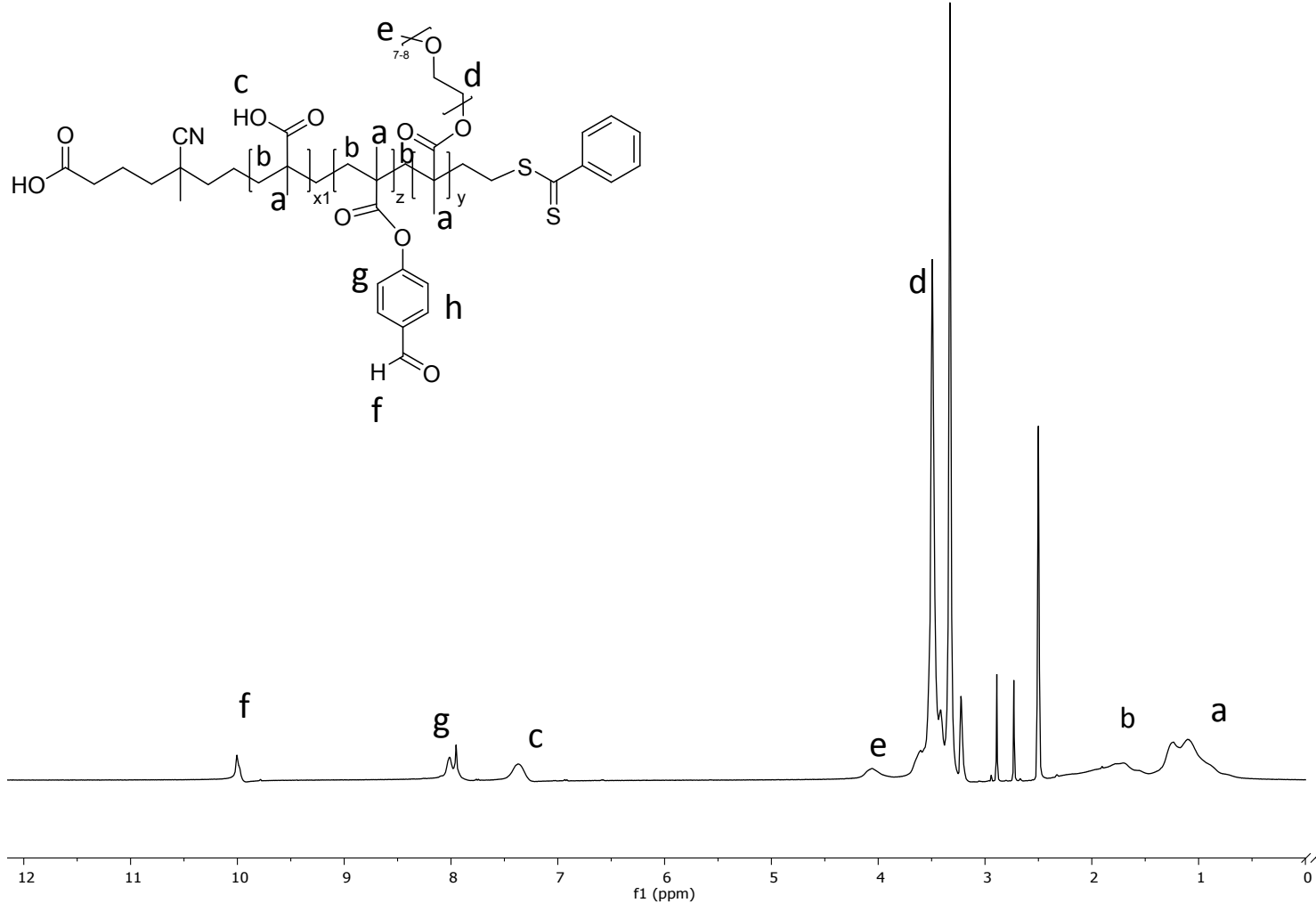

Figure S7: ${ }^{1} \mathrm{H}$ NMR spectrum of the post-functionalised copolymer with a 85:15 feed ratio.

$$
\begin{aligned}
& 100 * \frac{\operatorname{Integration}(e)}{3} /\left(\frac{\operatorname{Integration}(a)}{3}\right)=\text { OEGMA\% } \\
& 100 * \frac{\operatorname{Integration}(f)}{1} /\left(\frac{\operatorname{Integration}(a)}{3}\right)=B A \%
\end{aligned}
$$

$$
\left(\frac{\operatorname{Integration}(a)}{3}-\frac{\operatorname{Integration}(e)}{3}-\frac{\operatorname{Integration}(f)}{1}\right) * 100=\text { MAAc } \%
$$

substituting in numbers for 85:15 feed ratio:

Final Composition: poly(MAAc $\left.{ }_{60}-\mathrm{CO}-\mathrm{OEGMA} \mathrm{A}_{15}-\mathrm{CO}-\mathrm{BA}_{25}\right)$

Equation S3: Calculation of the post-functionalised copolymer (poly(MAAc-co-BA-co-OEGMA)) composition. A) Determines the OEGMA content by comparing the methyl protons of OEGMA to all of the methyl protons in the polymer backbone. B) The aldehyde protons are normalised against the polymer methyl protons and the ratio compared to determine the BA content. C) The BA and OEGMA contents are subtracted from the total methyl proton content, to determine the MAAc content.

Following the molecular weight conversion calculation in Equation $\mathrm{S} 2(26,500 \mathrm{~g} / \mathrm{mol})$, and by replacing $25 \%$ MAAc with BA (Equation S3), the approximate molecular weight can be calculated (Equation S4): 


$$
\begin{aligned}
& 0=M w_{T h}(85: 15)-M A A c(\text { feed })-O E G M A(\text { Feed }) \text { (a) } \\
& 0=26500-85 * 86 * 2 x-500 * 15 * 2 x \\
& \text { where } x=\text { conversion ratio } \\
& x=0.895 \quad(c) \\
& M w(P F)=2 x * M w_{M A A C} * n_{M A A C}+2 x * M w_{B A} * n_{B A}+2 x * M w_{O E G M A} * n_{O E G M A}(\mathrm{~d}) \\
& =2 * 0.895 * 86 * 60+0.895 *(86+105.12) * 25+2 * 0.895 *(500) * 15(e) \\
& \text { Molecular weight }=31,200 \frac{\mathrm{g}}{\mathrm{mol}}
\end{aligned}
$$

Equation S4: Calculating the post-functionalised molecular weight from the monomer conversion calculation (Equation S2, S3). A) Determination of the conversion ratio by taking the molecular weight from Equation S2. B) Substitution of molecular weights and feed ratios for an 85:15 copolymer $C$ ) The conversion ratio, where $0<x<1.0$. D) Molecular weight formula taking the ratios from Equation S3. E) Substitution of the molecular weight, conversion ratios and molecular feed ratios from Equation $\mathrm{S3}$.
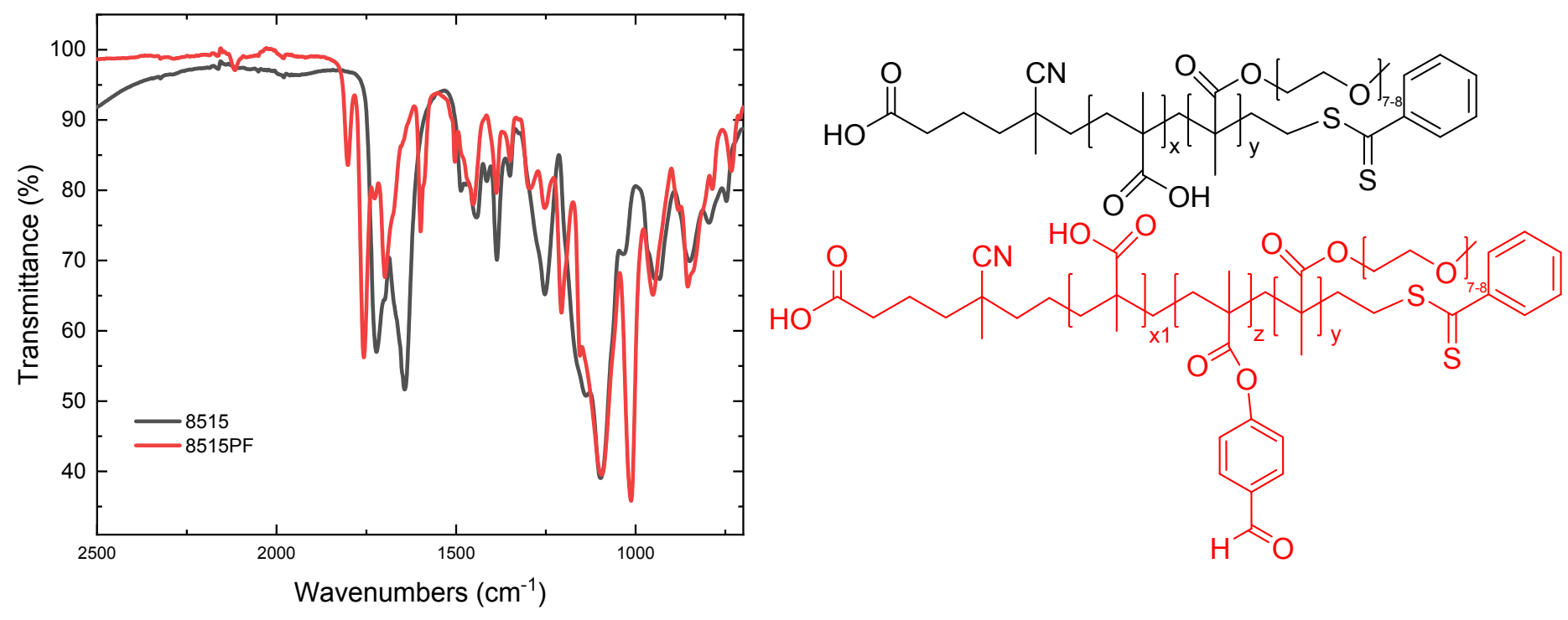

Figure S8: Zoomed FTIR spectrum of the post-functionalised 85:15 copolymer, with associated structures, colour coded (right). 


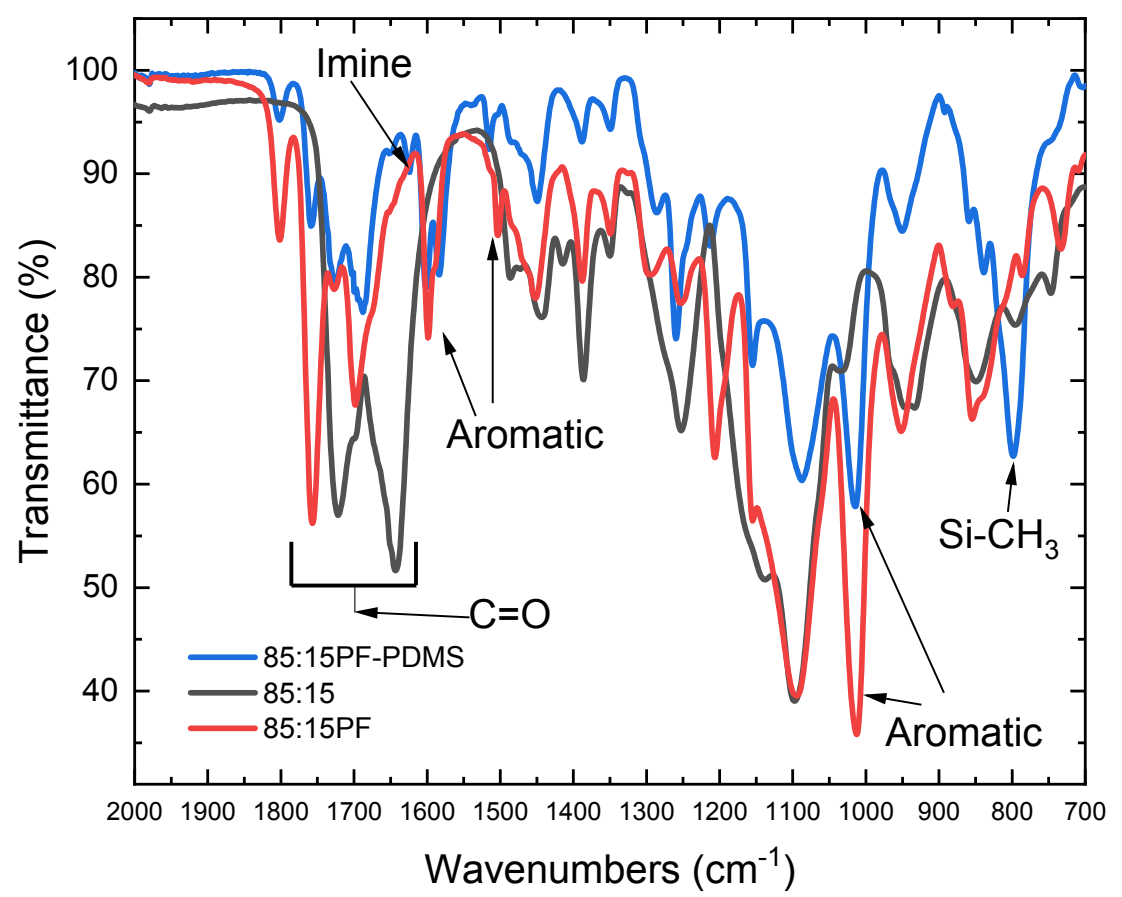

Figure S9: FTIR spectra snapshot of the copolymer (black), post-functionalised polymer (red) and a PDMS cross-linked post-functionalised polymer (blue). The snapshot highlights the formation of aromatic and imine bonds, as well as shifting of the carbonyl groups following functionalisation.

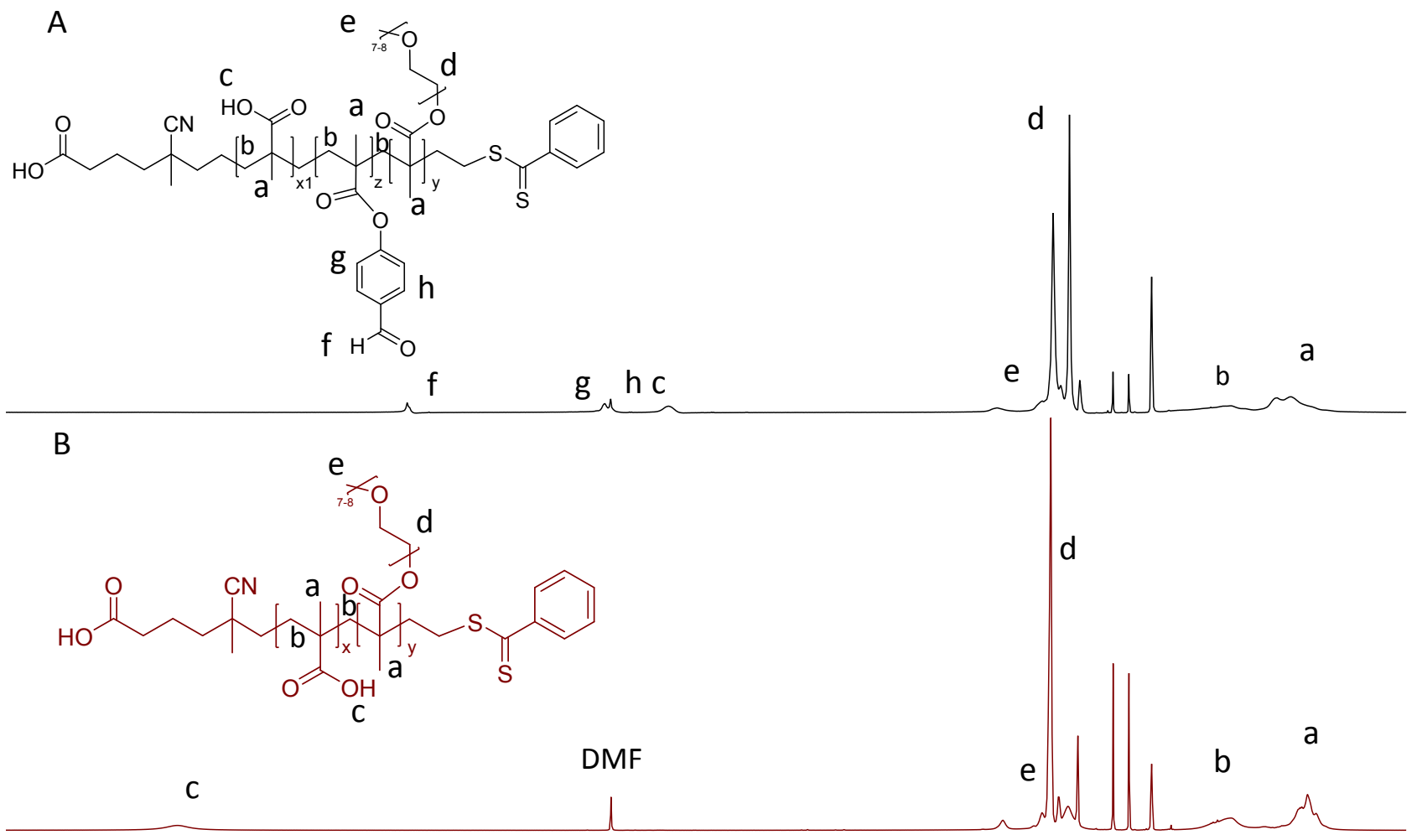

$\begin{array}{llllllllllllllllllllllllllllll}4.0 & 13.5 & 13.0 & 12.5 & 12.0 & 11.5 & 11.0 & 10.5 & 10.0 & 9.5 & 9.0 & 8.5 & 8.0 & 7.5 & \begin{array}{c}7.0 \\ \mathrm{f} 1 \mathrm{(pm})\end{array} & 6.5 & 6.0 & 5.5 & 5.0 & 4.5 & 4.0 & 3.5 & 3.0 & 2.5 & 2.0 & 1.5 & 1.0 & 0.5 & 0.1\end{array}$

Figure S10: ${ }^{1} \mathrm{H}$ NMR comparison of the copolymer to the post-functionalised polymer. A) ${ }^{1} \mathrm{H} N \mathrm{NR}$ spectrum of the ost-functionalised polymer (85:15PF, poly $\left(M A A c_{60}-\mathrm{BA}_{25}-O E G M A_{15}\right)$ ) following the 
esterification of benzaldehyde at the acid groups. The spectra highlights the formation off) aldehyde groups and g) aromatic regions. $B)^{1} H$ NMR spectrum of poly(MAAC $\left.{ }_{85}-\mathrm{CO}-O E G M A_{15}\right)$. 


$$
\frac{m(85: 15 P F)}{n(85: 15 P F)} * 200 * X L * 0.01 * M w(P D M S)=m(P D M S)
$$

Equation S5: Cross-linking density formula for determining the mass of the PDMS cross-linker, where $X L$ is the desired cross-linking density.
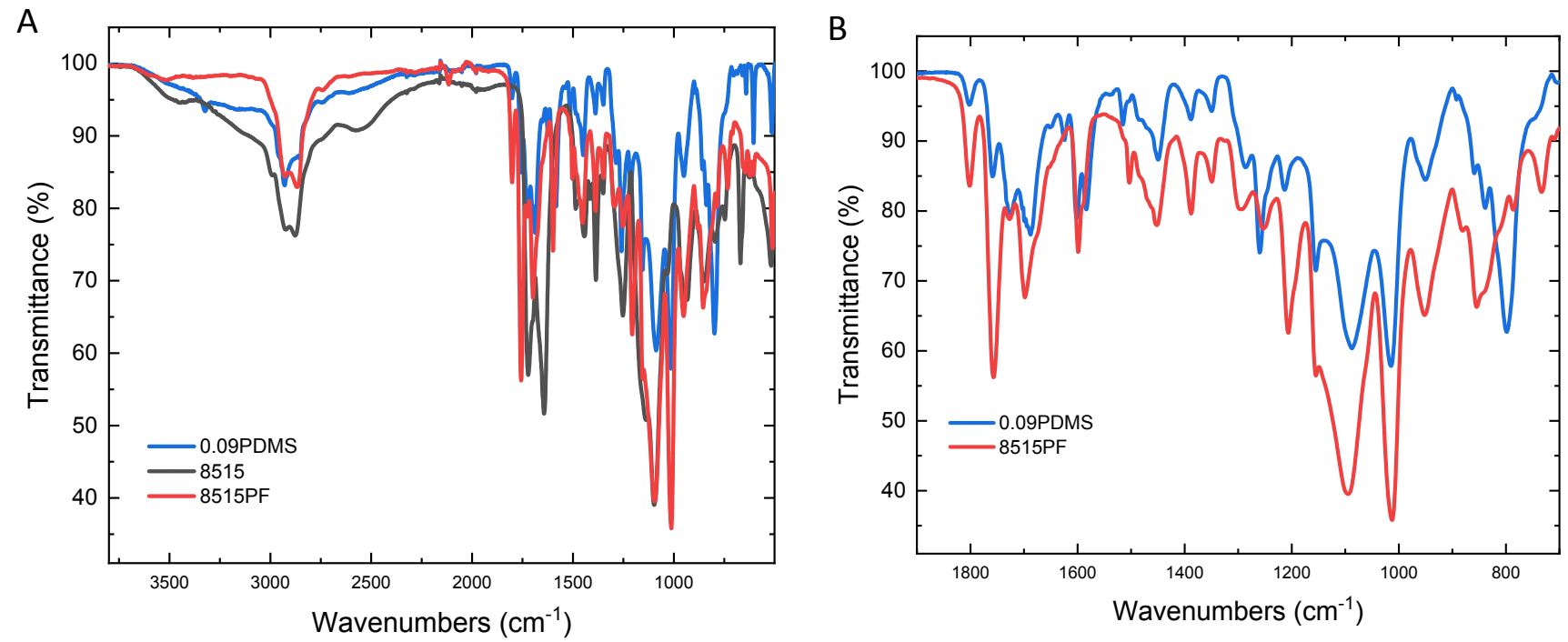

Figure S11: A: FTIR Spectrum of all three gels produced: 85:15 cross-linked with PDMS, 85:15 postfunctionalised and 85:15 copolymer. B: Zoomed in FTIR spectra of cross-linked and non-crosslinked $85: 15 \mathrm{ge} / \mathrm{s}$. Stretches at $800 \mathrm{~cm}^{-1}, 1600 \mathrm{~cm}^{-1}$ and $1750 \mathrm{~cm}^{-1}$ are notable additions in the cross-linked gels' spectrum. 


\subsection{Differential Scanning Calorimetry}
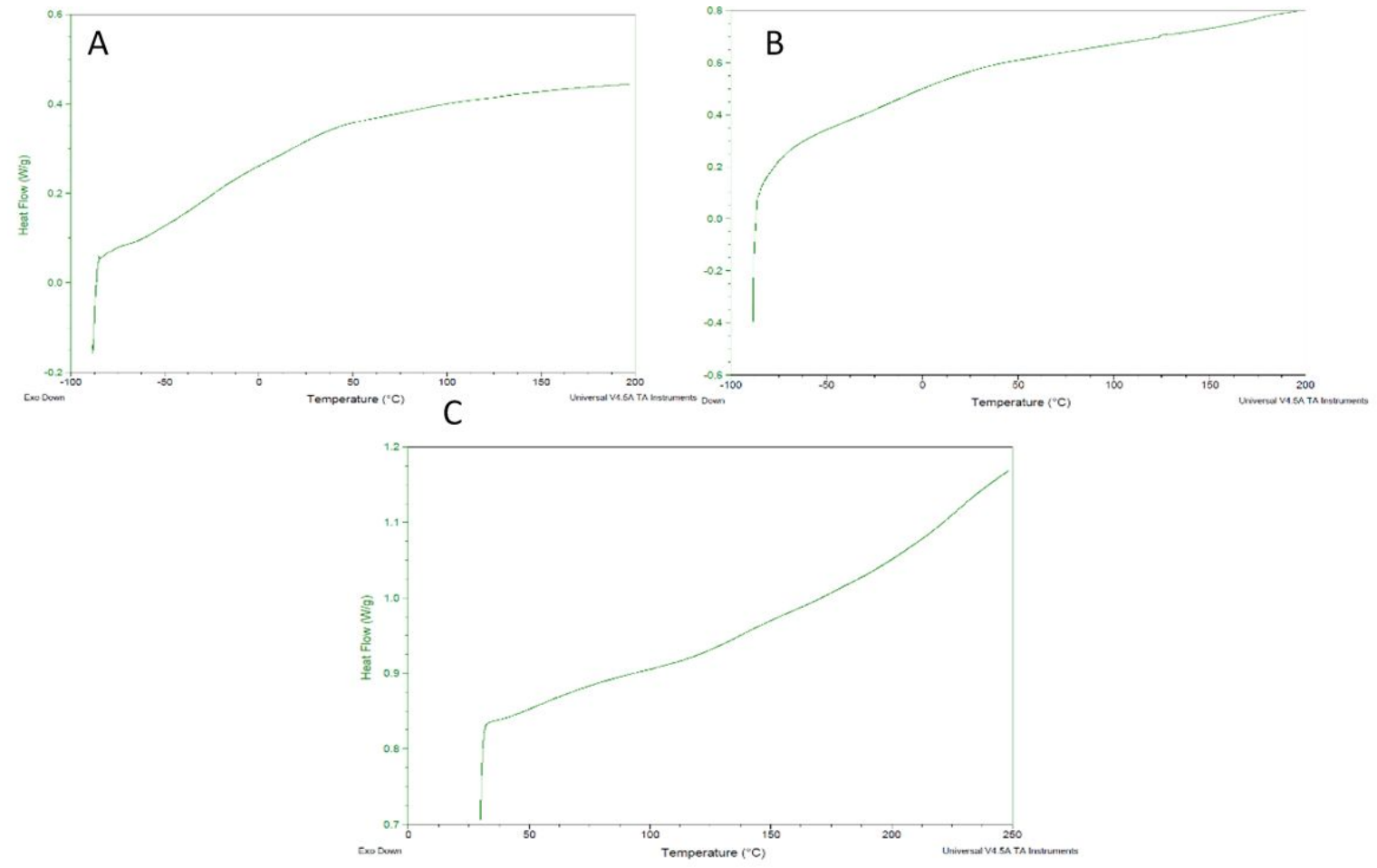

Figure S12 - DSC traces of the gels produced. A: 85:15 copolymer (Scheme 1). B: 85:15PF-0.12PDMS (Scheme 3). C: 85:15PF (Scheme 2). Note that all traces are from the second heating run to remove any heat memory or crystallization peaks. There are no peaks visible $(A-C)$. 


\subsection{Dynamic Mechanical Analysis}

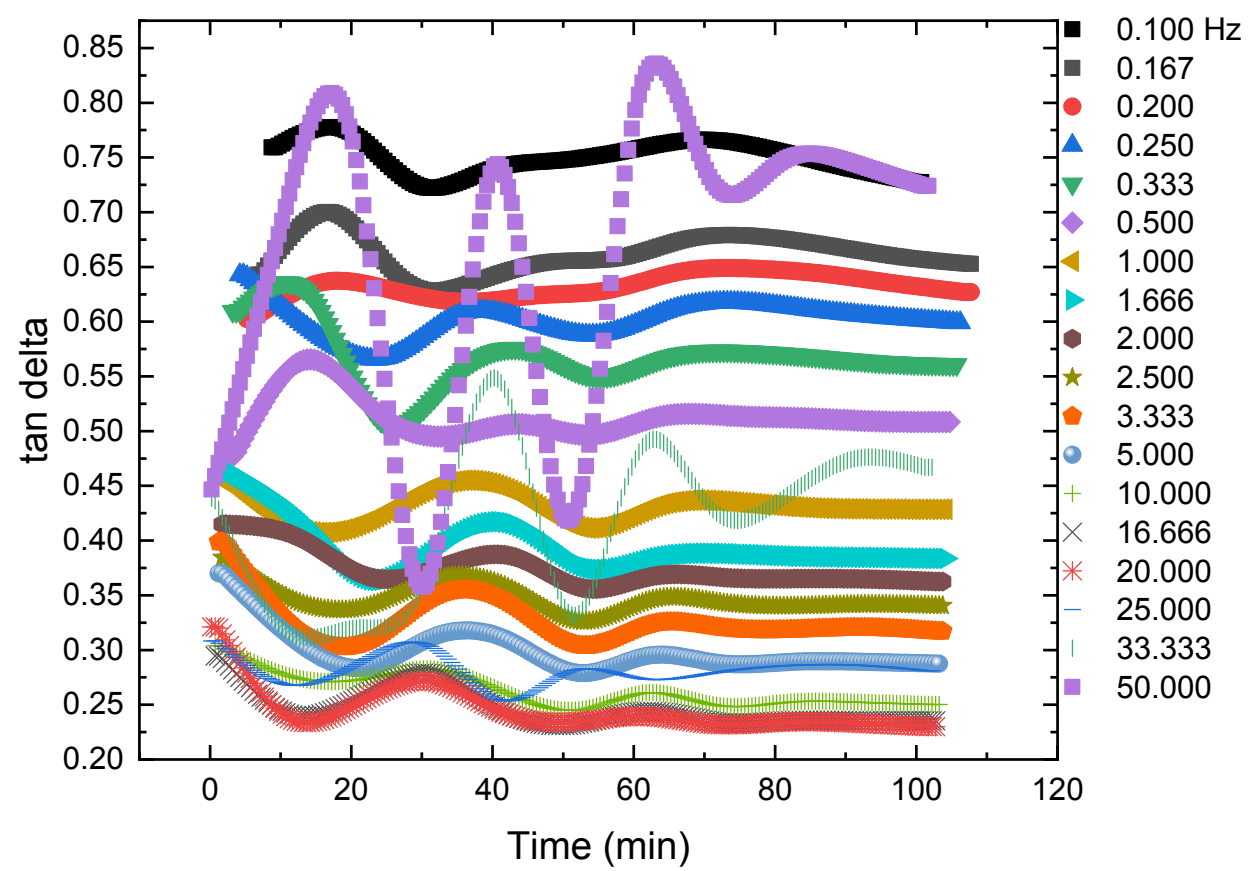

Figure S13: Frequency sweep of poly(MAAc-Co-BA-CO-OEGMA)-0.01PDMS, demonstrating how the tan delta curves reach equilibrium over time. The legend is the frequencies tested in $\mathrm{Hz}$.
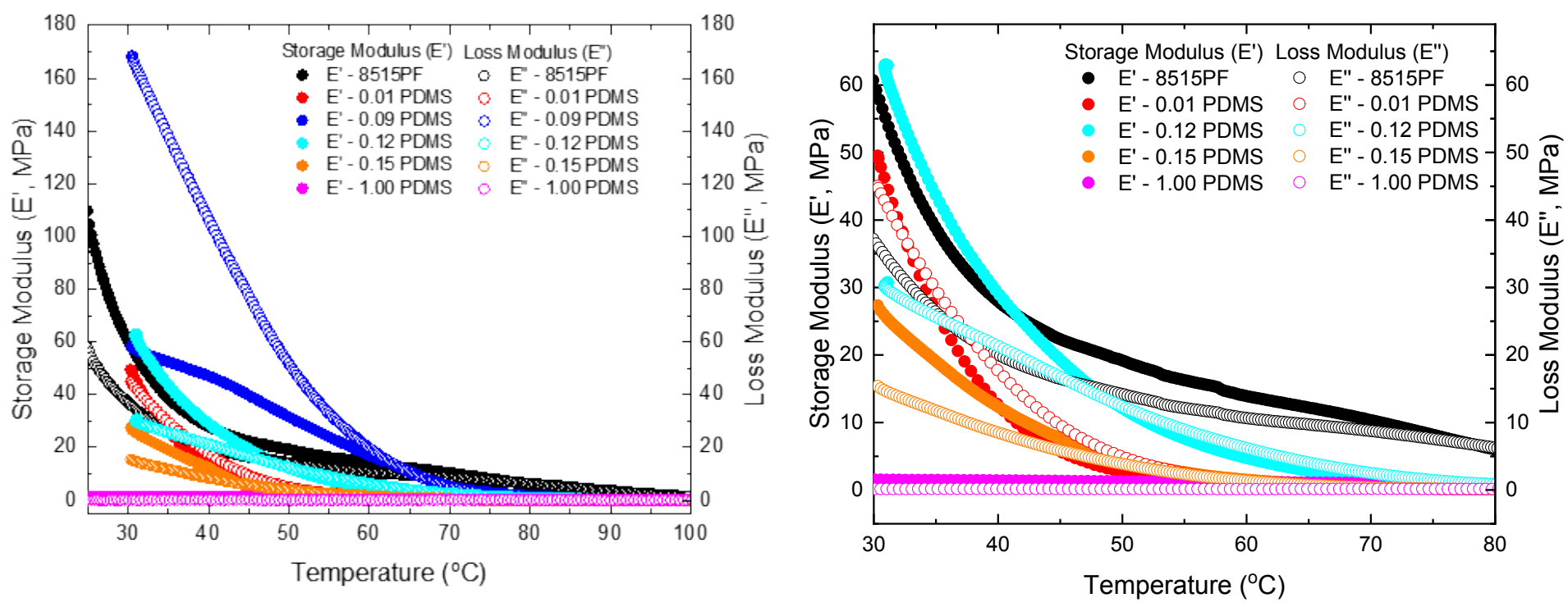

Figure S14: The change in storage and loss moduli as a function of temperature, across different compositions via Dynamic Mechanical Analysis. A: Full range of temperature and gels tested through dynamic mechanical analysis. B: Zoomed in spectra of select cross-linking densities. 


\subsection{Mechanical Testing}

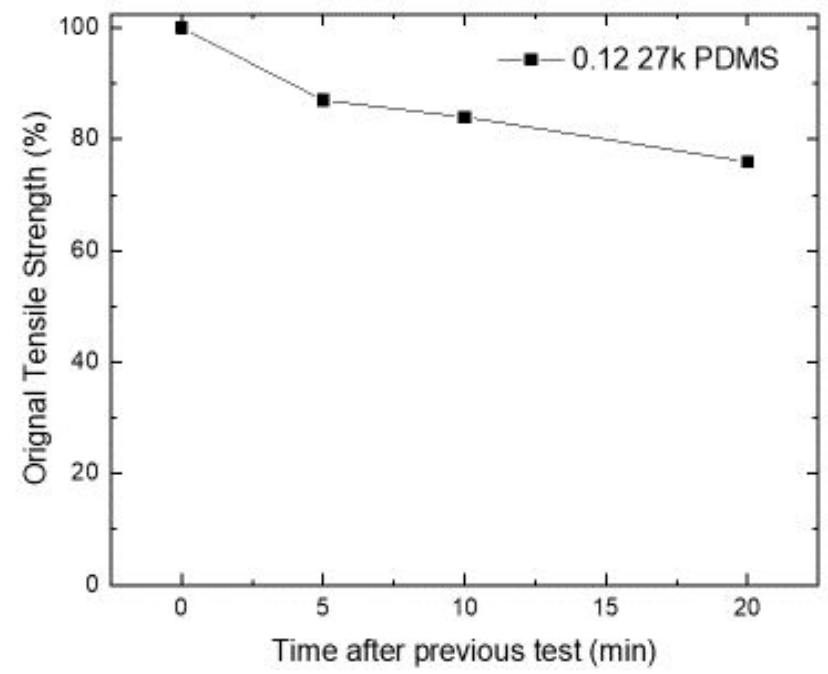

Figure S15: Cyclic loading and unloading demonstrating fatigue resistance of poly $\left(M A A c_{60}-\mathrm{CO}^{-} B A_{25^{-}}\right.$ co-OEGMA $A_{15}$ )-0.12PDMS gel. Through tensile testing, a hysteresis curve was constructed by stretching the gel to $50 \%$ of its breaking point. This occurred four times, with each subsequent test being further apart. The three tests were consecutive, 5 minute gap, 10 minute gap and then a 20 minute gap. These experiments were not continued due to DMA being becoming available.

i) Copolymer

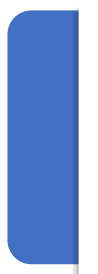

ii) Post-functionalised Gel

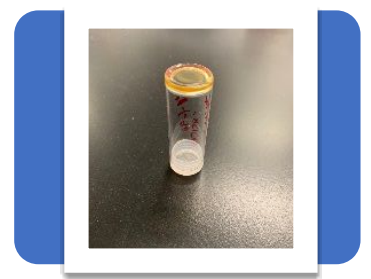

iii) PDMS Gel

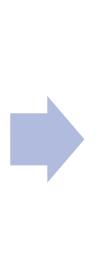

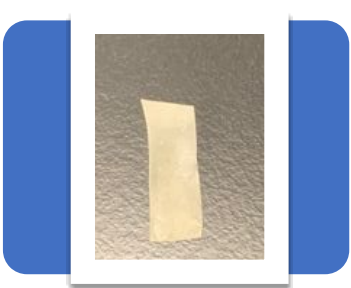

Figure S16: Photographical progression of the gel preperation process. The photographs typify the colour change of the dominating benzaldehyde over the RAFT agent from the transition of pink to yellow in the copolymer (i) to post-fucntionalised polymer (ii). The gel becomes more opaque and offwhite when PDMS is added (iii). The gel is also able to be thermally reversed at different temperatures. 


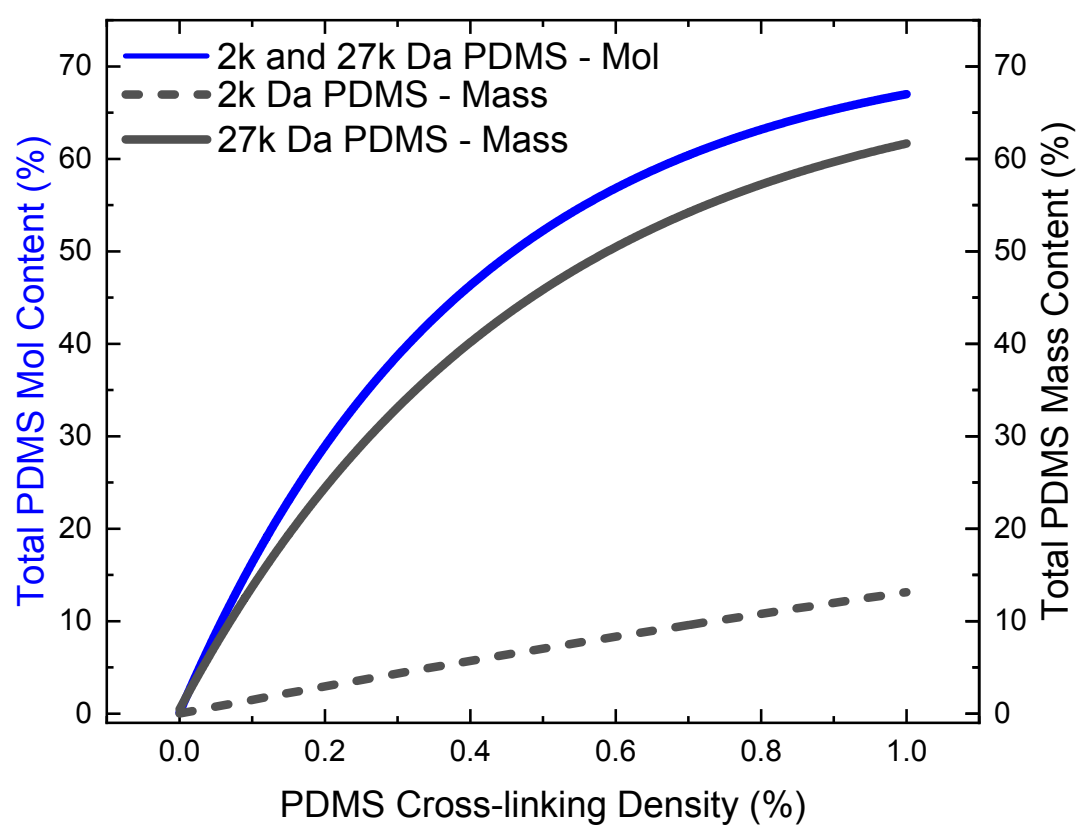

Figure S17: Relationship of the cross-linking density against the total mol and mass \% Content of PDMS in the entire network for $27 \mathrm{k}$ Da PDMS. The total mol \% content is a comparison of the moles of PDMS as a mol fraction of the post-functionalised copolymer plus the PDMS (Equation 2), (blue line). The total mass \% content compares the mass fraction of PDMS of the total nework for both $2 k$ and 27k Da PDMS (black dashed and filled lines, respectively; right hand axis). The cross-linking density (Supporting Information Equation S5) is a ratio of the number of aldehydes against the number of available acid groups.

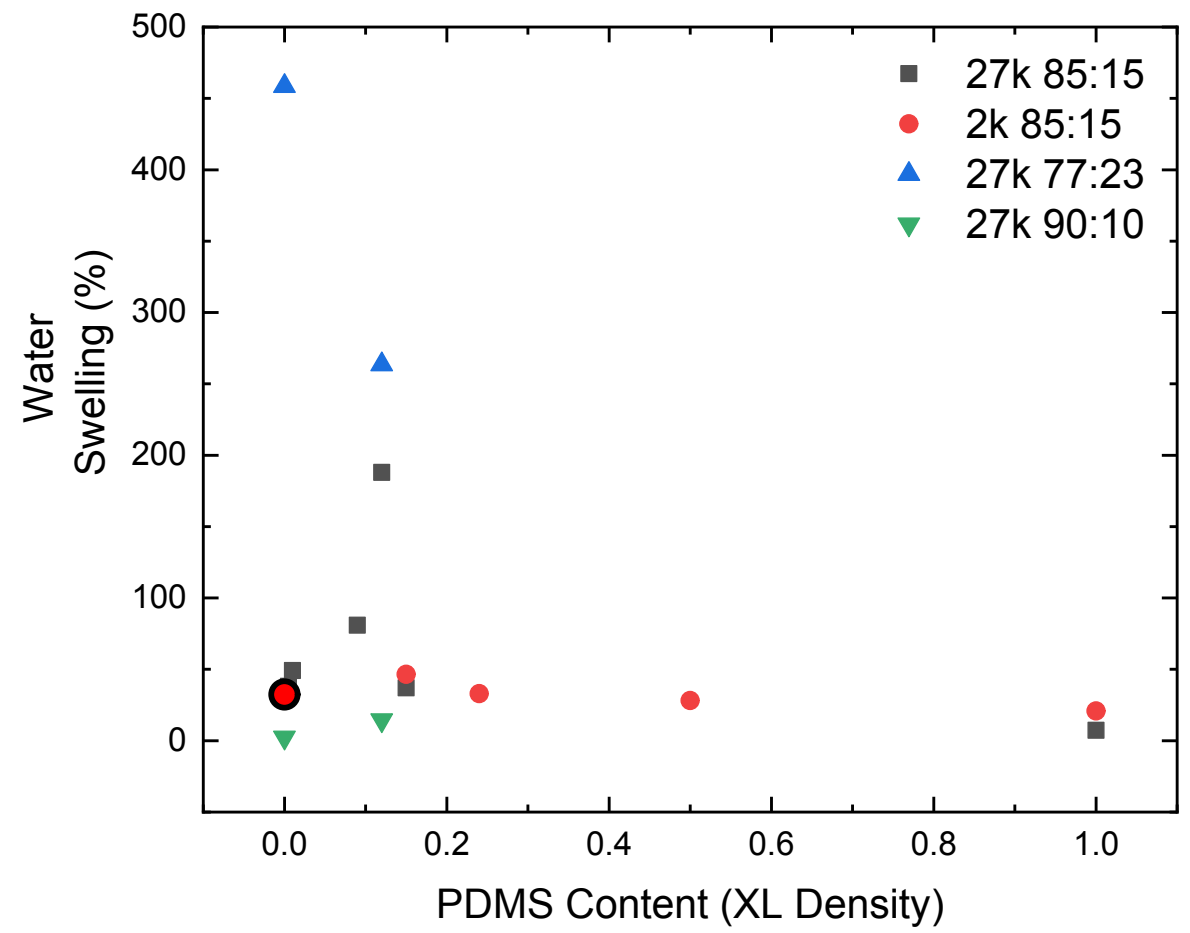

Figure S18: Modification of the PDMS content and the effect on the water swelling amount. The water swelling amount followed $24 \mathrm{~h}$ immersion in water and the water swelling percentage calculated from Equation 3. All samples were post-functionalised. The composition of the copolymers were also varied, with the PDMS cross-linker molecular weight notated immediately preceding the MAAc:OEGMA ratio noted in the legend (e.g. 77:23 =MAAc:OEGMA). 


$$
\text { Water Content }(\%)=\frac{m_{s}-m_{d}}{m_{d}} * 100
$$

Equation S6 - Equilibrium water swelling of hydrogels. Where $m_{s}$ is the mass in the swollen state, and $m_{d}$ is the mass in the dried state.

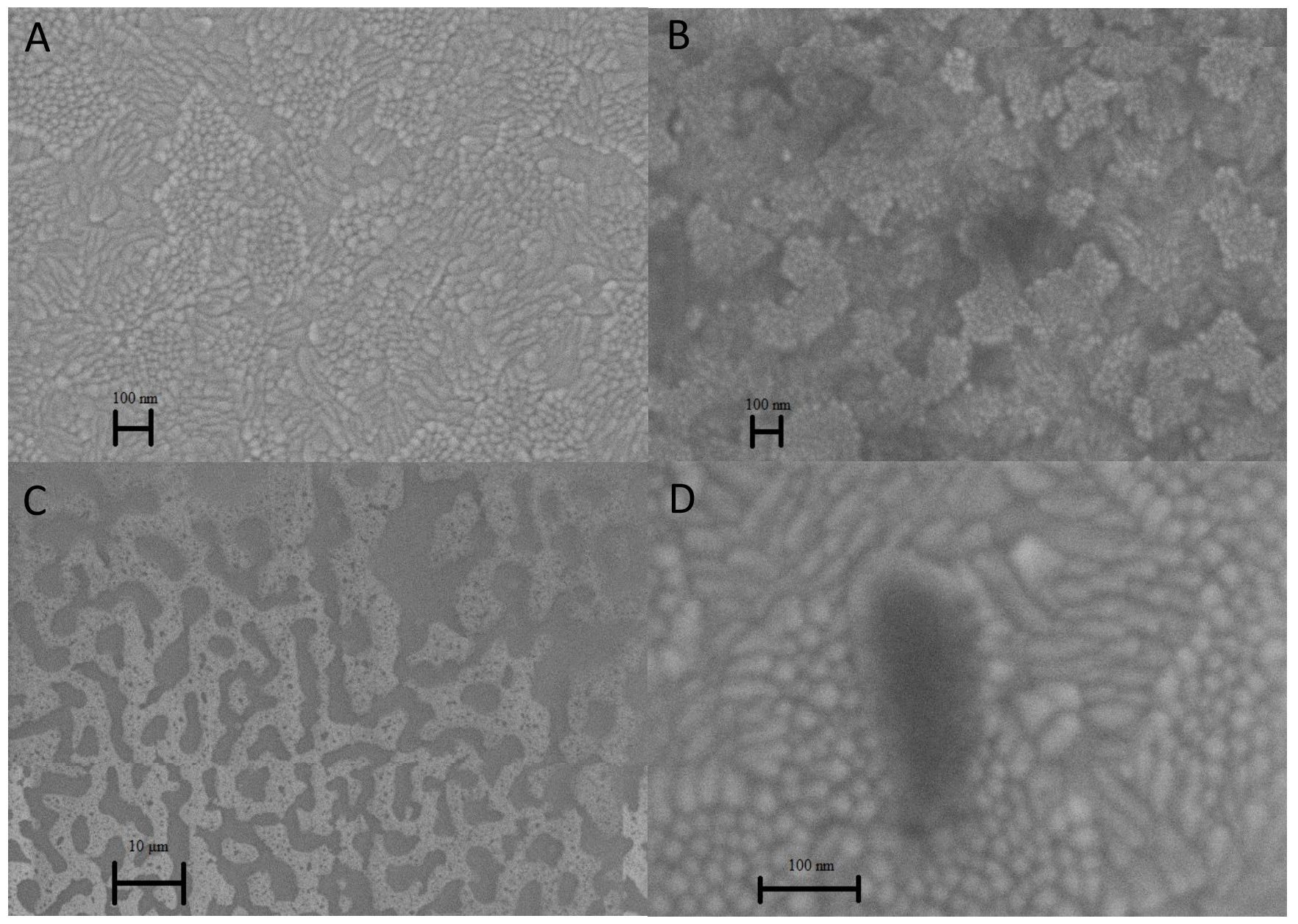

Figure S19 - Scanning Electron Microscope images. A: 85:15 Post-functionalised polymer with no cross-linker. Scale bars:100 nm. B: 1.00PDMS cross-linked polymer. Scale bars: 100nm. C: 1.00 PDMS cross-linked polymer macro-image. Scale bars of 10 um. D: Pure PDMS. Scale bars: $100 \mathrm{~nm}$. 


$$
\int_{\mathbf{0}}^{\epsilon_{f}} \sigma d \varepsilon=\frac{\text { Energy }}{\text { Volume }}=\frac{J}{m^{3}}
$$

Equation S7 - Mechanical definition of toughness, where $\sigma$ is the tensile strength, and $\varepsilon$ is the tensile strain.

Video S1: Video from the light microscope of the scratching of the gel with scissors and then the observed self-healing (shown in seminar). 